\title{
The hypergeometric series for the partition function of the 2-D Ising model
}

\author{
G. M. Viswanathan \\ Department of Physics and National Institute of Science and Technology of Complex \\ Systems, Universidade Federal do Rio Grande do Norte, 59078-970 Natal-RN, Brazil
}

\begin{abstract}
In 1944 Onsager published the formula for the partition function of the Ising model for the infinite square lattice. He was able to express the internal energy in terms of a special function, but he left the free energy as a definite integral. Seven decades later, the partition function and free energy have yet to be written in closed form, even with the aid of special functions. Here we evaluate the definite integral explicitly, using hypergeometric series. Let $\beta$ denote the reciprocal temperature, $J$ the coupling and $f$ the free energy per spin. We prove that $-\beta f=\ln (2 \cosh 2 K)-\kappa^{2}{ }_{4} F_{3}\left[{ }^{1,1,3 / 2,3 / 2} 2,2,2{ }_{2} \kappa^{2}\right]$, where ${ }_{p} F_{q}$ is the generalized hypergeometric function, $K=\beta J$, and $2 \kappa=\tanh 2 K \operatorname{sech} 2 \mathrm{~K}$.
\end{abstract}

PACS numbers: 05.50.+q 02.30.Gp 64.60.De 75.10.Hk

Journal version:

http://dx.doi.org/10.1088/1742-5468/2015/07/P07004

Submitted to: Journal of Statistical Mechanics (JSTAT) 
The Ising model was originally proposed by Lenz to describe magnetism [1]. His student Ising solved the eponymous model in one dimension (1-D) in his doctoral thesis. Since then, it has become one of the most important models in the history of statistical physics [2 7]. The 2-D case for zero external magnetic field was not fully solved until 1942, and the results were published only in 1944 [8]. The 3-D case remains unsolved. Onsager's derivation of the partition function of the 2-D Ising model is often described as a mathematical tour de force. He was able to express the internal energy in terms of a special function, but he left the partition function and the free energy in terms of a definite integral [8]. Ever since, a common assumption has been that there is no succinct way to write the partition function in closed form, even using special functions. The required integral is indeed not easy to evaluate explicitly, but we show that it is possible to do so. In fact, Onsager had already expanded the free energy in a power series [8], however he did not recognize it as a known special function (at least not until late in his life, see below). Here we continue the calculation where he left off and show that his power series is hypergeometric.

Readers unfamiliar with special functions may question what is gained by trading a definite integral for a special function. One is just as complicated as the other, it could be argued. Consider the following helpful analogy with trigonometric functions. For $|x| \leq 1$ the integral

$$
\int_{0}^{x} \frac{d y}{\sqrt{1-y^{2}}}
$$

can be written as $\arcsin x$. Neither expression contains more information than the other. Yet most readers will agree that $\arcsin x$ is preferrable to the integral, mainly because trigonometric functions are well understood. The same is true of special functions. In fact, the Hungarian mathematician Paul Turán thought that "special functions" should instead be renamed useful functions, according to Askey (see refs. [9, 10]). They are ubiquitous and arise in a variety of physical problems. For example, hypergeometric functions appear very naturally in mathematical physics [12 15], statistical mechanics [16, 17], and number theory [18].

We briefly review the square lattice Ising model with symmetric coupling. Consider a two dimensional square lattice where at each point $i$ of the lattice is located a spin-1/2 particle. Each spin $\sigma_{i}$ can assume only 2 values: $\sigma_{i}= \pm 1$. The Ising model Hamiltonian as a function of a spin configuration $\sigma=\left(\sigma_{1}, \sigma_{2}, \ldots\right)$ is given by

$$
H(\sigma)=-J \sum_{\langle i, j\rangle} \sigma_{i} \sigma_{j} .
$$

Here $\langle i, j\rangle$ represents the set of lattice points $i, j$ which are nearest neighbors. The sum should avoid double counting, so that pairs $\langle i, j\rangle$ and $\langle i, j\rangle$ are not counted separately. The constant $J$ is known as the coupling and is an interaction energy.

We are interested in the thermodynamic limit, but let us initially consider the partition function for a finite $L \times L$ system with $N=L^{2}$ spins. Let $T$ be the thermodynamic temperature, $k_{\mathrm{B}}$ Boltzmann's constant and let $\beta=1 /\left(k_{\mathrm{B}} T\right)$. The 
partition function $Z_{N}(\beta)$ is then defined as the sum over all possible spin configurations of $\exp (-\beta H(\sigma))$ :

$$
Z_{N}(\beta)=\sum_{\sigma} e^{-\beta H}
$$

So $Z_{N}$ can also be thought of as the two-sided Laplace transform of the degeneracy $\Omega(E)$ of the energy level $E$. The free energy is given by $F=-k_{\mathrm{B}} T \ln Z_{N}$ and the free energy per spin in the thermodynamic limit is given by $f=-k_{\mathrm{B}} T \ln \lambda$, where,

$$
\lambda=\lim _{N \rightarrow \infty} Z_{N}^{1 / N} .
$$

Onsager referred to $\lambda$ as the "partition function per atom" and henceforth we will refer to $\lambda$ simply as the partition function. In the seminal work of 1944, he derived the exact partition function,

$$
\begin{aligned}
\ln \lambda= & \ln 2 \cosh (2 K) \\
& +\frac{1}{2 \pi^{2}} \int_{0}^{\pi} \int_{0}^{\pi} \ln \left(1-4 \kappa \cos \omega_{1} \cos \omega_{2}\right) d \omega_{1} d \omega_{2},
\end{aligned}
$$

where

$$
2 \kappa=\tanh (2 K) \operatorname{sech}(2 K) .
$$

One of the two integrals can be evaluated, yielding

$$
\ln \left(\frac{\lambda}{2 \cosh 2 K}\right)=\frac{1}{2 \pi} \int_{0}^{\pi} \ln \left(\frac{1+\sqrt{\left(1-(4 \kappa \sin \varphi)^{2}\right.}}{2}\right) d \varphi
$$

and versions of expressions (44) and (6) are those found in the textbooks. For technical details and historical context, see refs. [1, 8, 19, 36].

Onsager wrote that the integral appearing in the partition function and free energy "cannot be expressed in closed form." It is true that the required closed form cannot be found among the commonly tabulated integrals, even in terms of special functions. Nevertheless, we will show below that it is certainly possible to express the integral in terms of a special function. Whether or not it is of "closed form" is a matter of context and convention. Traditionally, the term "closed form" does not include special functions. However, Onsager himself considered some special functions to be in closed form, for example the elliptic integrals (see [8] and the discussion below). Indeed, in the context of the Ising model and statistical mechanics, many special functions form part of the repertoire of "closed form" expressions. From this point of view, our result represents an advance. Before we state our claim, we review a few more relevant facts.

Although the free energy has never before been expressed in terms of special functions, yet it is possible [8] to express the internal energy per spin, defined by

$$
u=-\frac{\partial}{\partial \beta} \ln \lambda
$$

in terms of an elliptic integral:

$$
u=-J(\operatorname{coth} 2 K)\left(1+\frac{2}{\pi}\left(2(\tanh 2 K)^{2}-1\right) \mathrm{K}(4 \kappa)\right) \text {. }
$$


Here we have chosen the sans serif letter $\mathrm{K}$ to distinguish the complete elliptic integral of the first kind $\mathrm{K}(k)$ from the reduced reciprocal temperature $K=\beta J$. We use the same notation for the elliptic integral adopted in [8,9,37, where the argument $k$ of $\mathrm{K}(k)$ is the elliptic modulus and not the parameter (the latter defined as $m=k^{2}$ ). There are several conventions in use, so this is an important point to note. The definition is thus

$$
\mathrm{K}(k)=\int_{0}^{\pi / 2} \frac{d \theta}{\sqrt{1-(k \sin \theta)^{2}}} .
$$

Elliptic integrals can be expressed in terms of ${ }_{p} F_{q}$ generalized hypergeometric functions [9]. For example, the complete elliptic integral of the first kind above is equivalently given by

$$
\mathrm{K}(k)=\left(\frac{\pi}{2}\right){ }_{2} F_{1}\left[\begin{array}{c}
\frac{1}{2} \frac{1}{2} ; k^{2} \\
1
\end{array}\right] \text {. }
$$

We briefly explain this notation. $\mathrm{A}_{p} F_{q}$ function has a power series $\sum c_{n} x^{n}$ such that the ratio $c_{n+1} / c_{n}$ of successive coefficients is a rational function of $n$, i.e. a ratio of polynomials in the degree $n$ of the summed monomials. The numbers $p$ and $q+1$ give the degrees of the polynomials of the numerator and denominator, respectively. Let the Pochhammer symbol $(x)_{n}$ denote the rising factorial,

$$
\begin{aligned}
& (x)_{0}=1 \\
& (x)_{n}=(x)_{n-1}(x+n-1) ; \quad n=1,2,3 \ldots
\end{aligned}
$$

Equivalently, in terms of the gamma function $\Gamma(x)$, the Pochhammer symbol is given by $(x)_{n}=\Gamma(x+n) / \Gamma(x)$. The ${ }_{p} F_{q}$ function is then concisely defined as follows:

$$
{ }_{p} F_{q}\left[\begin{array}{c}
a_{1}, a_{2}, \ldots, a_{p} \\
b_{1}, b_{2}, \ldots, b_{q}
\end{array} ; x\right]=\sum_{n=0}^{\infty} \frac{\left(a_{1}\right)_{n}\left(a_{2}\right)_{n} \ldots\left(a_{p}\right)_{n}}{\left(b_{1}\right)_{n}\left(b_{2}\right)_{n} \ldots\left(b_{q}\right)_{n}} \frac{x^{n}}{n !} .
$$

The condition $p=q+1$ separates the two distinct regimes $p<q+1$ for which the ${ }_{p} F_{q}$ function is entire, and $p>q+1$ when the radius of convergence is zero. When $p=q+1$ exactly, the radius of convergence is 1 (with convergence on the unit circle a somewhat delicate issue). In our case, we will find $p=4$ and $q=3$ so that $p=q+1$. Similarly, for the elliptic integral in (8) we can see from (10) that $p=2$ and $q=1$. So these functions have unit radius of convergence. The singularity of $\mathrm{K}(4 \kappa)$ at $4 \kappa=1$ in Eq. (8) is precisely what is responsible for critical point of the phase transition in the 2-D Ising model. The radius of convergence corresponds to the celebrated critical temperature of the phase transition, first found by Kramers and Wannier [21] in 1941.

Having reviewed the necessary definitions, we state our main result:

Theorem 1 Onsager's partition function $\lambda$ in (4) can be written in terms of a hypergeometric function as $\lambda_{*}$, where

$$
\ln \lambda_{*}=\ln (2 \cosh 2 K)-\kappa^{2}{ }_{4} F_{3}\left[\begin{array}{cc}
1,1, \frac{3}{2}, \frac{3}{2} & ; 16 \kappa^{2} \\
2,2,2 &
\end{array}\right],
$$

and where $\kappa$ is defined by (5). 
The ${ }_{4} F_{3}$ function above cannot be reduced to a sum of functions of simpler type. For example, comparing series one can show that the following ${ }_{3} F_{2}$ function is reducible in terms of simpler functions:

$$
{ }_{3} F_{2}\left[1, \frac{3}{2}, \frac{3}{2} ; 2,2 ; x\right]=-\frac{4}{x}+\frac{8}{\pi x} \mathrm{~K}\left(x^{1 / 2}\right) .
$$

In contrast, the ${ }_{4} F_{3}$ in Theorem 1 cannot be reduced in this manner, i.e. it cannot be expressed as a sum of functions of simpler type (see also ref. [39]).

The ${ }_{4} F_{3}$ hypergeometric function in Theorem 1 is not entirely unexpected in fact, because the integral in (44) is of a type known as a Mahler measure [12, 13, that can lead to ${ }_{4} F_{3}$ functions. The Mahler measure associated with the integral in Onsagers formula can be obtained from Eq. (39) in ref. [11, Eq. (17) in ref. [12] or Eq. (12) in ref. [13].

We have very recently learnt [38] that, in the 1970s, Glasser and Onsager working together arrived at a similar (but different) expression to the one above in Theorem 1 . They used $\mathrm{a}_{4} F_{3}$ function as well as the complete elliptic integral of the second kind, $\mathrm{E}(k)$. However, they did not publish their result. Considering the potentially broad interest in this fascinating piece of historical information, below we restate their previously unpublished result.

Theorem 2 (Glasser and Onsager) The partition function $\lambda$ in (4) can be rewritten as $\lambda_{\star}$, where

$$
\begin{aligned}
\ln \lambda_{\star}=\ln (2 \cosh 2 K) & \\
& -\frac{1}{2}+\frac{1}{\pi} \mathrm{E}(4 \kappa)+\kappa^{2}{ }_{4} F_{3}\left[\begin{array}{c}
\frac{1}{2}, 1,1, \frac{3}{2} ; 16 \kappa^{2} \\
2,2,2
\end{array}\right] .
\end{aligned}
$$

Below we give rigorous proofs of Theorems 1 and 2, but first we briefly discuss the intuition and method behind the discovery. The logarithm inside the integral in Eq. (4) can be expanded in a Taylor series. One can perform the definite integral term by term and then sum them up. Onsager himself performed this calculation and arrived at the following expression for the partition function:

$$
\ln \lambda=\ln (2 \cosh 2 K)-\sum_{n=1}^{\infty}\left(\begin{array}{c}
2 n \\
n
\end{array}\right)^{2} \frac{\kappa^{2 n}}{4 n} .
$$

He did not proceed further, except to note the finite radius of convergence.

We instead approached the sum in (15) as a formal power series. Unlike normal power series, formal power series are defined algebraically, independently of convergence. Rather than interpreting this series analytically as converging to a function, we instead attempted to match the coefficients in the series with those in the formal power series definitions of special functions. If all coefficients match, then the two series are equal in an algebraic sense and we will have found the desired special function. Theorems 1 and 2 in fact follow from (15).

It is interesting to note that although Eqs (44) and (15) above appear together as Eq. (109c) in Onsager's 1944 paper, yet (4) is widely known whereas (15) has received very 
little attention in comparison. When we recently came across (15) for the first time, we immediately suspected that the series was a ${ }_{p} F_{q}$ generalized hypergeometric function. Even with only passing familiarity with special functions, readers will recognize the following clues pointing to a generalized hypergeometric function: (i) a power series, (ii) factorials in the numerator and denominator of the series coefficients and (iii) the arguments of the factorials grow with the degree of the monomials.

Onsager himself seems to have been at least partially aware of the connection with hypergeometric functions, for he wrote in the appendix,

We shall deal here with the evaluation of various integrals which occur in the text. Most of these can be reduced in straightforward fashion to complete elliptic integrals; only the partition function itself is of a type one step higher than the theta functions, [emphasis added] and involves a little analysis which is not found in textbooks.

As mentioned earlier, the elliptic integrals are special cases of the ${ }_{2} F_{1}$ ordinary or Gaussian hypergeometric functions. Moreover, the theta and elliptic functions are related to elliptic integrals or their inverses. Onsager states that the partition function is "one step higher" than the theta functions, and indeed the ${ }_{4} F_{3}$ function that we find in the evaluation of the partition function is a step or two more complicated than the ${ }_{2} F_{1}$ functions.

The power series method we originally used to arrive at (13) from (15) is purely algebraic. Moreover, it is possible to guarantee the correct behavior on and outside the radius of convergence. One way around the convergence issue is analytic continuation. However, this is in fact not needed because the series converges even at the critical temperature and can be evaluated in terms of Catalan's constant. We will not further discuss these technical points and will instead take a much easier-to-understand approach.

We give below an elementary proof. Noting that differentiation is much simpler than integration, we will differentiate $-\ln \lambda_{*}$ and then use the fundamental theorem of calculus, obtaining $u$. So $\ln \lambda$ and $\ln \lambda_{*}$ differ only by a constant, which we will show is zero. We next state and prove a hypergeometric identity, from which Theorem 1 follows easily:

\section{Lemma 1}

$$
\begin{aligned}
{ }_{4} F_{3} & {\left[\begin{array}{c}
2,2, \frac{5}{2}, \frac{5}{2} \\
3,3,3
\end{array}\right] x } \\
& =-\frac{128}{9 x^{2}}+\frac{256 \mathrm{~K}(\sqrt{x})}{9 \pi x^{2}}-\frac{32}{9 x}{ }_{4} F_{3}\left[\begin{array}{c}
1,1, \frac{3}{2}, \frac{3}{2} ; x \\
2,2,2
\end{array}\right] .
\end{aligned}
$$

Proof Using the series definition (12), the term of degree $n \geq-1$ of $\left({ }_{4} F_{3}\left[\begin{array}{c}2,2, \frac{5}{2}, \frac{5}{2} \\ 3,3,3\end{array} ; x\right]+\right.$ 
$\left.\frac{32}{9 x}{ }_{4} F_{3}\left[\begin{array}{c}1,1, \frac{3}{2}, \frac{3}{2} \\ 2,2,2\end{array} ; x\right]\right)$ is

$$
\begin{aligned}
& \frac{(2)_{n}^{2}\left(\frac{5}{2}\right)_{n}^{2}}{n !(3)_{n}^{3}} x^{n}+\frac{32}{9 x} \frac{(1)_{n+1}^{2}\left(\frac{3}{2}\right)_{n+1}^{2}}{(n+1) !(2)_{n+1}^{3}} x^{n+1} \\
& =\frac{128}{9 \pi}\left(\frac{\Gamma\left(\frac{5}{2}+n\right)}{\Gamma(3+n)}\right)^{2} x^{n} .
\end{aligned}
$$

Similarly, for the expression $\frac{256}{9 \pi x^{2}} \mathrm{~K}(\sqrt{x})$, which by (10) is $\left(\frac{256}{18 x^{2}}\right)_{2} F_{1}\left[\begin{array}{c}1 / 2,1 / 2 \\ 1\end{array} ; x\right]$, we get for $n \geq-2$ :

$$
\frac{256}{18 x^{2}} \frac{(1 / 2)_{n+2}^{2}}{(n+2) !(1)_{n+2}} x^{n+2}=\frac{128}{9 \pi}\left(\frac{\Gamma\left(\frac{5}{2}+n\right)}{\Gamma(3+n)}\right)^{2} x^{n} .
$$

The two series thus agree for the coefficients of $x^{n}$ for every $n \geq-1$, but for $n=-2$ the ${ }_{4} F_{3}$ terms do not contribute. The lone $x^{-2}$ term, for $n=-2$ in the above expression, is

$$
\frac{128}{9 \pi x^{2}}\left(\frac{\Gamma\left(\frac{5}{2}-2\right)}{\Gamma(3-2)}\right)^{2}=\frac{128}{9 x^{2}} \text {. }
$$

This term cancels the term $-\frac{128}{9 x^{2}}$ in (16) and the claim follows.

Proof of Theorem 1 Observe from (7) that $-\ln \lambda$ is an antiderivative of $u$. Recall that the infinitely many antiderivatives $\int u(\beta) d \beta$ of the internal energy $u(\beta)$ differ only by a constant. Our strategy will be to show that $-\ln \lambda_{*}$ is also an antiderivative of $u$ and with the same integration constant.

The general formula for the derivative of a ${ }_{4} F_{3}$ function is

$$
\begin{aligned}
& \frac{d}{d x}{ }_{4} F_{3}\left[\begin{array}{c}
a_{1}, a_{2}, a_{3}, a_{4} \\
b_{1}, b_{2}, b_{3}
\end{array}\right] x \\
& \quad=\frac{a_{1} a_{2} a_{3} a_{4}}{b_{1} b_{2} b_{3}} F_{3}\left[\begin{array}{c}
a_{1}+1, a_{2}+1, a_{3}+1, a_{4}+1 \\
b_{1}+1, b_{2}+1, b_{3}+1
\end{array}\right] x .
\end{aligned}
$$

This general differentiation rule gives us

$$
\frac{d}{d x}{ }_{4} F_{3}\left[\begin{array}{c}
1,1, \frac{3}{2}, \frac{3}{2} \\
2,2,2
\end{array} ; x\right]=\frac{9}{32}{ }_{4} F_{3}\left[\begin{array}{c}
2,2, \frac{5}{2}, \frac{5}{2} \\
3,3,3
\end{array} ; x .\right.
$$

From the supporting lemma, we then get

$$
\begin{aligned}
& \frac{d}{d x}{ }_{4} F_{3}\left[\begin{array}{c}
1,1, \frac{3}{2}, \frac{3}{2} \\
2,2,2
\end{array} ; x\right] \\
& =-\frac{4}{x^{2}}+\frac{8 \mathrm{~K}(\sqrt{x})}{\pi x^{2}}-\frac{{ }_{4} F_{3}\left[\begin{array}{c}
1,1, \frac{3}{2}, \frac{3}{2} \\
2,2,2
\end{array} ; x\right]}{x} .
\end{aligned}
$$


This result together with the product rule gives us

$$
\frac{d}{d x}\left(x_{4} F_{3}\left[\begin{array}{c}
1,1, \frac{3}{2}, \frac{3}{2} \\
2,2,2
\end{array} ; x\right]\right)=-\frac{4}{x}+\frac{8 \mathrm{~K}(\sqrt{x})}{\pi x} .
$$

Notice that we get cancellation of the terms with the ${ }_{4} F_{3}$ functions, leaving only the elliptic integral and an elementary term. We point out that this result can also be understood in terms of an order-four linear differential operator that factors in terms of lower order operators, see ref. [39].

We will differentiate $\ln \lambda_{*}$ starting from (13) using the chain rule. Note, however, that $\kappa$ as a function of $\beta$ is not invertible, because $\kappa(\beta)$ is not monotonic in $\beta$ and so the inverse function $\beta(\kappa)$ is multivalued. To get around this problem, let us introduce the change of variable $\operatorname{arctanh} \mathrm{v}=2 \mathrm{~K}$, so that

$$
\begin{aligned}
\tanh 2 K=(\operatorname{coth} 2 K)^{-1} & =v \\
\cosh 2 K=(\operatorname{sech} 2 \mathrm{~K})^{-1} & =\frac{1}{\sqrt{1-v^{2}}} \\
\sinh 2 K=(\operatorname{csch} 2 \mathrm{~K})^{-1} & =\frac{v}{\sqrt{1-v^{2}}} \\
\kappa=\frac{1}{2} \tanh 2 K \operatorname{sech} 2 \mathrm{~K} & =\frac{1}{2} v \sqrt{1-v^{2}} .
\end{aligned}
$$

The hyperbolic function $\cosh 2 K$ and the quantity $\kappa$ in (13) can be eliminated by re-expressing them in terms of the new variable $v$. Hence, direct substitution of (24) into (13) gives us,

$$
\begin{aligned}
\ln \lambda_{*}= & \ln \frac{2}{\sqrt{1-v^{2}}} \\
& -\frac{v^{2}\left(1-v^{2}\right)}{4}{ }_{4} F_{3}\left[\begin{array}{c}
1,1, \frac{3}{2}, \frac{3}{2} ; 4 v^{2}\left(1-v^{2}\right) \\
2,2,2
\end{array}\right] .
\end{aligned}
$$

We now use (23) to calculate $d \ln \lambda_{*} / d v$ using the chain rule for derivatives. We get, after simplification,

$$
\frac{\partial \ln \lambda_{*}}{\partial v}=\frac{\pi-\left(2-4 v^{2}\right) \mathrm{K}\left(2 v \sqrt{1-v^{2}}\right)}{2 \pi v\left(1-v^{2}\right)} .
$$

Note how the differentiation of $\ln \lambda_{*}$ thus leads to a remarkable reduction of type ${ }_{4} F_{3}$ to type ${ }_{2} F_{1}$. We next use the chain rule again:

$$
\frac{\partial \ln \lambda_{*}}{\partial \beta}=\frac{\partial \ln \lambda_{*}}{\partial v} \frac{\partial v}{\partial K} \frac{\partial K}{\partial \beta}=2\left(1-v^{2}\right) J \frac{\partial \ln \lambda_{*}}{\partial v} .
$$

From (26) and (27) we get the following final expression:

$$
-\frac{\partial \ln \lambda_{*}}{\partial \beta}=-\frac{J}{v}\left(1+\frac{2}{\pi}\left(2 v^{2}-1\right) \mathrm{K}\left(2 v \sqrt{1-v^{2}}\right)\right) .
$$

Observe that (28) and (8) are identical, after changing variables using (24). We have thus shown that $-\ln \lambda_{*}(\beta)$ is an antiderivative of $u(\beta)$. We are almost done. 
Since $-\ln \lambda_{*}$ and $-\ln \lambda$ are both antiderivatives of $u$ with respect to $\beta$, therefore by the fundamental theorem of calculus they differ only by a real constant. Let $C=\ln \lambda-\ln \lambda_{*}$. To show equality of $\lambda_{*}$ and $\lambda$, it suffices to show that $C=0$. We can calculate $C$ explicitly from the values of both $\lambda_{*}$ and $\lambda$ for some convenient value of $\beta$. The easiest choice is $\beta=0$, for which we get $\ln \lambda_{*}(0)=\ln \lambda(0)=\ln 2$. So $C=0$ and the claim follows.

Proof of Theorem 2 We will proceed as with Lemma 1 and derive a hypergeometric identity, from which Theorem 2 will follow immediately.

On the one hand, the coefficient of the term of degree $\kappa^{2 n}$ for all $n \geq 1$ of the quantity

$$
\kappa_{4}^{2} F_{3}\left[\begin{array}{c}
1,1, \frac{3}{2}, \frac{3}{2} \\
2,2,2
\end{array} ; 16 \kappa^{2}\right]
$$

is

$$
16^{n-1} \frac{(1)_{n-1}^{2}\left(\frac{3}{2}\right)_{n-1}^{2}}{(n-1) !(2)_{n-1}^{3}}=\frac{4^{2 n-1}}{\pi n^{3}}\left(\frac{\Gamma\left(n+\frac{1}{2}\right)}{\Gamma(n)}\right)^{2} .
$$

On the other hand, the quantity

$$
\frac{1}{\pi} \mathrm{E}(4 \kappa)+\kappa^{2}{ }_{4} F_{3}\left[\begin{array}{c}
\frac{1}{2}, 1,1, \frac{3}{2} \\
2,2,2
\end{array} ; 16 \kappa^{2}\right]
$$

can be rewritten completely in terms of hypergeometric functions as

$$
\left(\frac{1}{2}\right){ }_{2} F_{1}\left[\begin{array}{c}
\frac{1}{2},-\frac{1}{2} ; 16 \kappa^{2} \\
1
\end{array}\right]+\kappa^{2}{ }_{4} F_{3}\left[\begin{array}{c}
\frac{1}{2}, 1,1, \frac{3}{2} ; 16 \kappa^{2} \\
2,2,2
\end{array}\right] .
$$

The term of degree zero in the series above is $1 / 2$ because only the ${ }_{2} F_{1}$ term contributes. For all $n \geq 1$ the term of degree $2 n$ has coefficient

$$
\begin{aligned}
& \frac{1}{2} \frac{\left(\frac{1}{2}\right)_{n}\left(-\frac{1}{2}\right)_{n}}{n !(1)_{n}}+16^{n-1} \frac{\left(\frac{1}{2}\right)_{n-1}(1)_{n-1}^{2}\left(\frac{3}{2}\right)_{n-1}}{(n-1) !(2)_{n-1}^{3}} \\
& =-\frac{4^{2 n-1}}{\pi} \frac{\Gamma\left(n-\frac{1}{2}\right) \Gamma\left(n+\frac{1}{2}\right)}{[\Gamma(n+1)]^{2}}+\frac{2^{4 n-3}}{\pi n^{3}} \frac{\Gamma\left(n-\frac{1}{2}\right) \Gamma\left(n+\frac{1}{2}\right)}{[\Gamma(n)]^{2}} \\
& =-\frac{4^{2 n-1}}{\pi n^{3}}\left(\frac{\Gamma\left(n+\frac{1}{2}\right)}{\Gamma(n)}\right)^{2} .
\end{aligned}
$$

Observing that (29) and (32) are identical except for sign for all $n \geq 1$ and then taking into account separately the previously mentioned $n=0$ term, we arrive at

$$
\kappa^{2}{ }_{4} F_{3}\left[\begin{array}{c}
1,1, \frac{3}{2}, \frac{3}{2} \\
2,2,2
\end{array} ; 16 \kappa^{2}\right]=\frac{1}{2}-\frac{1}{\pi} \mathrm{E}(4 \kappa)-\kappa^{2}{ }_{4} F_{3}\left[\begin{array}{c}
\frac{1}{2}, 1,1, \frac{3}{2} \\
2,2,2
\end{array} ; 16 \kappa^{2}\right] .
$$

The claim follows from substitution of this hypergeometric identity into Theorem 1 , 
The hypergeometric series for the partition function of the 2-D Ising model

\section{Acknowledgments}

We thank T. M. Viswanathan for discussions and for suggesting the study of formal power series. We thank J. C. Cressoni and S. Salinas for feedback and CNPq for funding. We are grateful to the anonymous referees for helpful suggestions.

\section{References}

[1] Ising E., Beitrag zur Theorie des Ferromagnetismus, 1925 Z. Physik 31253

[2] Stanley H E, 1971 Introduction to Phase Transitions and Critical Phenomena (Oxford: Oxford University Press)

[3] Feynman R P, 1972 Statistical Mechanics. A set of lectures (Reading: Benjamin and Cummings Publishing)

[4] McCoy B and Wu T, 1973 The two dimensional Ising model (Cambridge: Harvard University Press)

[5] Huang K, 1987 Statistical Mechanics (New York John Wiley \& Sons)

[6] Baxter R J 1989 Exactly solved models in statistical mechanics (London: Academic Press)

[7] Salinas S, 2001 Introduction to Statistical Physics (Berlin: Springer)

[8] L. Onsager, Crystal Statistics. I. A Two-Dimensional Model with an Order-Disorder Transition, 1944 Phys. Rev. 65117

[9] Andrews G E, Askey R, and Roy R, Special functions, 1999 Encyclopedia of Mathematics and Its Applications 71, Ed. G. C. Rota (Cambridge: Cambridge University Press)

[10] Cipra B A, A new testament for special functions? 1998 SIAM News 31(2)

[11] Rogers M, Hypergeometric Formulas for Lattice Sums and Mahler Measures 2011 IMRN 174027

[12] Guttmann A J and Rogers M D, Spanning tree generating functions and Mahler measures, 2012 J. Phys. A 45494001 (arXiv:1207.2815 [math-ph])

[13] Glasser M L, A note on a hyper-cubic Mahler measure and associated Bessel integral, $2012 \mathrm{~J}$. Phys. A 45494002

[14] Glasser M L and Lamb G, A lattice spanning-tree entropy function, 2005 J. Phys A: Math. Gen. 38, L471

[15] Joyce G S Application of Mahler measure theory to the face-centred cubic lattice Green function at the origin and its associated logarithmic integral, 2012 J. Phys. A: Math. Theor. 45285001

[16] Simmons J J H, Kleban P, Flores S M and Ziff R M, Cluster densities at 2D critical points in rectangular geometries, 2011 J. Phys. A: Math. Theor. 44385002

[17] Assis M, Boukraa S, Hassani S, van Hoeij M, Maillard J.-M and McCoy B M Diagonal Ising susceptibility: elliptic integrals, modular forms and Calabi-Yau equations, 2012 J. Phys. A: Math. Theor. 45, 075205

[18] Rogers M D, New ${ }_{5} F_{4}$ hypergeometric transformations, three-variable Mahler measures, and formulas for $\frac{1}{\pi}$, 2009 Ramanujan J. 18327

[19] Longuet-Higgins H C and Fisher M E, Lars Onsager: November 27, 1903October 5, 1976, 1995 J. Stat. Phys. 78, 605

[20] Peierls, R On Ising's Model of Ferromagnetism, 1936 Proc. Cambridge Phil. Soc. 32477

[21] Kramers H A and Wannier G H, Statistics of the two-dimensional ferromagnet, 1941 Physical Review 60252

[22] van der Waerden B L Die lange Reichweite der regelmäßigen Atomanordnung in Mischkristallen, 1941 Z. Physik 118473

[23] Kac M and J. C. Ward J C A Combinatorial Solution of the Two-Dimensional Ising Model, 1952 Phys. Rev. $\mathbf{8 8} 1332$

[24] Sherman S, Combinatorial Aspects of the Ising Model for Ferromagnetism. I. A Conjecture of Feynman on Paths and Graphs, 1960 J. Math. Phys. 1202 
[25] Hurst C A and H. S. Green H S, New Solution of the Ising Problem for a Rectangular Lattice J. Chem. Phys. 331059 (1960).

[26] Temperley H N V and Fisher M E, Dimer problem in statistical mechanics-an exact result, 1961 Phil. Mag. 61061

[27] Kasteleyn P W The statistics of dimers on a lattice: I. The number of dimer arrangements on a quadratic lattice, 1961 Physica 271209

[28] Burgoyne P N, Remarks on the combinatorial approach to the Ising problem, $1963 \mathrm{~J}$. Math. Phys. 41320

[29] Vdovichenko N V, Spontaneous magnetization of a plane dipole lattice, 1965 Soviet Physics JETP 20477

[30] Brush S G, History of the Lenz-Ising Model 1967 Rev. Mod. Phys. 39883

[31] Newell G F and Montroll E W, On the Theory of the Ising Model of Ferromagnetism, 1953 Rev. Mod. Phys. 25, 353

[32] Baxter R J and Enting I G, 399th solution of the Ising model, 1978 J. Phys. A 112463

[33] Cipra B A, An introduction to the Ising model 1987 The American Mathematical Monthly 94937

[34] da Costa G A T F and Maciel A L, Combinatorial Formulation of Ising Model Revisited, 2003 Rev. Bras. Ensino de Fsica 2549

[35] Schultz T D, Mattis D C and Lieb E H, Two-dimensional Ising model as a soluble problem of many fermions, 1964 Rev. Mod. Phys. 36856

[36] McCoy B M, The Romance of the Ising Model, 2011 arXiv:1111.7006 [math-ph]

[37] Whittaker E T and Watson G N, 1902 A course of modern analysis (Cambridge: Cambridge University Press)

[38] Glasser M L, personal e-mail communication, 12 November 2014

[39] Boukraa S, Hassani S, Maillard J-M, McCoy B M, Weil J-A and and Zenine N, Fuchs versus Painlevé, 2007 J. Phys. A 4012589 